ANNALES

POLONICI MATHEMATICI

$87(2005)$

\title{
Positively homogeneous functions and the Kojasiewicz gradient inequality
}

\author{
by Alain Haraux (Paris)
}

To the memory of Stanistaw Eojasiewicz

\begin{abstract}
It is quite natural to conjecture that a positively homogeneous function with degree $d \geq 2$ on $\mathbb{R}^{N}$ satisfies the Eojasiewicz gradient inequality with exponent $\theta=1 / d$ without any need for an analyticity assumption. We show that this property is true under some additional hypotheses, but not always, even for $N=2$.
\end{abstract}

Introduction. The starting point of this work is convergence to equilibrium of global bounded solutions of the first order gradient system governed by the equation

$$
u^{\prime}+\nabla F(u)=0, \quad t \geq 0 .
$$

The following basic result, generalized in many recent papers [1-7, 12] to various finite- or infinite-dimensional gradient-like dynamical systems, has been obtained by S. Łojasiewicz [8, 10]:

THEOREM 0.1. Let $F: U \subset \mathbb{R}^{N} \rightarrow \mathbb{R}$ be real analytic with $U$ open and let $u$ be any bounded global solution of (1.1) with the closure of its range contained in $U$. There exists $a \in \mathcal{E}=\{a \in U: \nabla F(a)=0\}$ such that

$$
\lim _{t \rightarrow+\infty}\|u(t)-a\|=0 .
$$

The proof of Theorem 0.1 relies on the following deep result also due to Łojasiewicz $[8,9]$.

TheORem 0.2. Let $F: U \subset \mathbb{R}^{N} \rightarrow \mathbb{R}$ be real analytic and let $a \in \mathcal{E}$. There are two real numbers (depending on a in general) $\theta \in(0,1 / 2)$ and $\eta>0$ such that

$$
\forall u \in U, \quad\|u-a\| \leq \eta \Rightarrow\|\nabla F(u)\| \geq|F(u)-F(a)|^{1-\theta} .
$$

2000 Mathematics Subject Classification: 26B35, 26E05, 34A26, 34D05.

Key words and phrases: homogeneous functions, Łojasiewicz inequality. 
The proof of Theorem 0.2 is quite involved and is based on the theory of subanalytic sets. As observed by Łojasiewicz in his pioneering papers, in (0.3) the number $\theta$ can be replaced by any $\theta^{\prime}<\theta$. However recently in several papers (cf. e.g. $[4,5]$ ) it was observed that the case $\theta=1 / 2$ is also of some interest, corresponding to exponential convergence to equilibrium for solutions of (0.1). This corresponds to the best possible case for analytic functions. But generally, the upper bound of possible $\theta$ for (0.3) does not satisfy (0.3) any more. For the limiting value, (0.3) must be replaced by the perhaps less elegant, but still useful form

$$
\forall u \in U, \quad\|u-a\| \leq \eta \Rightarrow\|\nabla F(u)\| \geq \delta|F(u)-F(a)|^{1-\theta}
$$

for some $\delta>0$. In addition, as shown in [4], under condition (0.3) with $\theta<1 / 2$, all solutions of $(0.1)$ converging to $a$ at infinity are such that

$$
\forall t \geq 0, \quad\|u(t)-a\| \leq C t^{-\theta /(1-2 \theta)}
$$

for some $C>0$. Since $\theta /(1-2 \theta)$ is an increasing function of $\theta$, it is important in practice to know the best (highest) possible value for $\theta$ satisfying (0.4). In this paper, we concentrate on the case where $F$ is positively homogeneous of degree $d \geq 2$. A natural conjecture, based on the one-dimensional case, is that $\theta=1 / d$, even for some non-analytic functions $F$. The object of this paper is to discuss the validity of this conjecture.

1. Preliminary remarks. We recall that a function $F: \mathbb{R}^{N} \rightarrow \mathbb{R}$ is called positively homogeneous of degree $d>0$ if

$$
\forall u \in \mathbb{R}^{N}, \forall \lambda>0, \quad F(\lambda u)=\lambda^{d} F(u) .
$$

A positively homogeneous function is exactly determined by its trace on

$$
\Sigma=\left\{u \in \mathbb{R}^{N}:\|u\|=1\right\} .
$$

However, even if $F$ is smooth in the neighbourhood of $\Sigma$, the regularity of $F$ at 0 depends on other properties. For instance if $F \not \equiv 0, F$ cannot be differentiable at 0 if $d<1$. If in addition $F \geq 0$, the differentiability of $F$ at 0 requires $d>1$, in which case $\nabla F(0)=0$. In this paper we shall consider only the case $d \geq 2$. If in addition $F \in C^{1}\left(\mathbb{R}^{N}\right)$, we have

$$
\forall u \in \mathbb{R}^{N}, \forall \lambda>0, \quad \nabla F(\lambda u)=\lambda^{d-1} \nabla F(u) .
$$

Proposition 1.1. Let $F \in C^{1}\left(\mathbb{R}^{N}\right)$ be positively homogeneous with degree $d \geq 1$. Then for any ball $B$ centred at 0 , there exists $C=C(B)>0$ such that

$$
\forall u \in B, \quad|F(u)| \leq C\|\nabla F(u)\| .
$$

Proof. This is an immediate consequence of Euler's formula

$$
\forall u \in \mathbb{R}^{N}, \quad F(u)=\frac{1}{d}\langle\nabla F(u), u\rangle .
$$


Proposition 1.2. Let $F \in C^{1}\left(\mathbb{R}^{N}\right)$ be positively homogeneous of degree $d>1$. Assume that for some ball $B$ centred at 0 , there exists $C>0$ such that

$$
\forall u \in B, \quad|F(u)|^{1-\theta} \leq C\|\nabla F(u)\| .
$$

Then if $F \not \equiv 0$, we must have

$$
\theta \leq 1 / d
$$

Proof. Let $\xi \in \Sigma$ be such that $F(\xi) \neq 0$. We have

$$
\forall \lambda>0, \quad F(\lambda \xi)=\lambda^{d} F(\xi)
$$

and

$$
\forall \lambda>0, \quad \nabla F(\lambda \xi)=\lambda^{d-1} \nabla F(\xi) .
$$

Therefore (1.5) implies the existence of $M>0$ such that

$$
\lambda^{d(1-\theta)} \leq M \lambda^{d-1}
$$

as $\lambda \rightarrow 0$. This in turn implies $d(1-\theta) \geq d-1$, equivalent to (1.6).

2. The two-dimensional case. In this section we assume $N=2$. In polar coordinates $(r, \phi)$ any homogeneous function $F$ of degree $d$ can be represented by

$$
F(r, \phi)=r^{d} f(\phi)
$$

where $f$ is $2 \pi$-periodic in $\phi$. An immediate calculation gives

$$
\|\nabla F(r, \phi)\|=r^{d-1}\left(d^{2} f^{2}(\phi)+f^{\prime 2}(\phi)\right)^{1 / 2} .
$$

In order for $F$ to satisfy $(0.4)$ with $\theta=1 / d$, it is necessary and sufficient that for some constant $C>0$,

$$
|f(\phi)| \leq C\left(|f(\phi)|^{d /(d-1)}+\left|f^{\prime}(\phi)\right|^{d /(d-1)}\right)
$$

or equivalently

$$
\exists \alpha>0,|f(\phi)| \leq \alpha \Rightarrow|f(\phi)| \leq 2 C\left|f^{\prime}(\phi)\right|^{d /(d-1)},
$$

which can be rephrased by saying that $f$ must satisfy the Łojasiewicz inequality with exponent $1 / d$ in the neighbourhood of its zeroes.

The first main result of this section is the following

Theorem 2.1. Let $F$ be a homogeneous polynomial on $\mathbb{R}^{2}$ of degree $d \geq 2$. Then $F$ satisfies inequality (0.4) with $\theta=1 / d$.

Proof. We have in fact

$$
F(u)=F(x, y)=r^{d} F(\cos \phi, \sin \phi) .
$$


It is clear that $f(\phi)=F(\cos \phi, \sin \phi)$ has a finite number of zeroes modulo $2 \pi$. Since $F$ is homogeneous, we can also write, for $\phi \neq \pi / 2(\bmod \pi)$,

$$
F(\cos \phi, \sin \phi)=\cos ^{d} \phi P(\tan \phi)=\cos ^{d} \phi Q(\tan \phi) \prod_{j}\left(\tan \phi-a_{j}\right)^{m_{j}}
$$

where the polynomial $Q$ has a constant sign. Therefore outside any neighbourhood of the discrete set $\pi / 2+\mathbb{Z} \pi$ we have

$$
F(\cos \phi, \sin \phi)=R_{1}(\phi) \prod_{j}\left(\sin \phi-a_{j} \cos \phi\right)^{m_{j}}=R_{2}(\phi) \prod_{j}\left(\sin \left(\phi-\alpha_{j}\right)\right)^{m_{j}}
$$

where $R_{1}, R_{2}$ are non-vanishing functions and $\sum_{j} m_{j} \leq d$. Then as $\phi$ approaches one of the zeroes of $f$ not in $\pi / 2+\mathbb{Z} \pi$, a straightforward calculation shows that

$$
|f(\phi)| \leq C\left|f^{\prime}(\phi)\right|^{m_{j} /\left(m_{j}-1\right)} \leq C^{\prime}\left|f^{\prime}(\phi)\right|^{d /(d-1)} .
$$

A similar argument for the zeroes in $\pi / 2+\mathbb{Z} \pi$ follows from exchanging the roles of sin and $\cos$ (or $x$ and $y$ ).

Proposition 2.2. For any $d \in \mathbb{N}^{*}$ there exists a $C^{d-1}$ function, positively homogeneous of degree $d$ on $\mathbb{R}^{2}$, which does not satisfy (0.4) with $\theta=1 / d$.

Proof. Consider, for any integer $p>0$, the function

$$
F(x, y)=\frac{x^{p+d}}{\left(x^{2}+y^{2}\right)^{p / 2}} .
$$

It is clear that $F \in C^{d-1}\left(\mathbb{R}^{2}\right)$ with all partial derivatives up to order $d-1$ vanishing at the origin and $F$ is homogeneous of degree $d$. But we also have

$$
F(x, y)=r^{d} \sin ^{p+d} \phi
$$

so that clearly condition (2.4) is violated.

REMARK 2.3. Actually, since all partial derivatives of order $d-1$ are uniformly Lipschitz on the unit ball, the function $F$ given by (2.5) just fails to be $C^{d}\left(\mathbb{R}^{2}\right)$. It is on the other hand easy to see that if a $d$-homogeneous function is $C^{d}\left(\mathbb{R}^{2}\right)$, it is in fact a homogeneous polynomial of degree $d$ (cf. Proposition 5.1). As a consequence, both Proposition 2.2 and Theorem 2.1 are quite sharp. For a sharper result on the function $F$ given by (2.5), see Section 5 .

REMARK 2.4. The result of Theorem 2.1 is no longer valid in dimensions $N>2$. As we shall see in Section 3 it is valid for monomials in any dimension, and it is also easily checked to be true in any dimension for polynomials of degree 2 (quadratic forms). A "minimal" counterexample is therefore expected to have at least two terms of degree 3 and three variables. Now we consider

$$
P(x, y, z)=x^{2} y-z^{2} x .
$$


It is immediate to check that for any $\varepsilon>0$ fixed

$$
\forall t>0, \quad\left|P\left(\frac{1}{2 \varepsilon} t^{2}, \varepsilon, t\right)\right|=\frac{1}{4 \varepsilon} t^{4}
$$

whereas

$$
\forall t>0, \quad\left\|\nabla P\left(\frac{1}{2 \varepsilon} t^{2}, \varepsilon, t\right)\right\| \sim \frac{1}{\varepsilon} t^{3} \quad \text { as } t \rightarrow 0 .
$$

It follows immediately that here $\theta \leq 1 / 4<1 / 3=1 / d$.

3. Generalized monomials. Let $\left\{p_{j}\right\}_{1 \leq j \leq N}$ be a finite collection of exponents such that

$$
\forall j \in\{1, \ldots, N\}, \quad p_{j} \geq 1 .
$$

Let $\left\{\varepsilon_{j}\right\}_{1 \leq j \leq N}$ be a finite collection of exponents such that

$$
\forall j \in\{1, \ldots, N\}, \quad \varepsilon_{j} \in\{0,1\} .
$$

We consider the function

$$
F(u)=F\left(u_{1}, \ldots, u_{N}\right)=\prod_{1 \leq j \leq N}\left|u_{j}\right|^{p_{j}}\left(\operatorname{sgn} u_{j}\right)^{\varepsilon_{j}} .
$$

It is clear that $F$ is positively homogeneous of degree $d:=\sum_{1<j<N} p_{j}$ and absolutely continuous with

$$
|F(u)|=\prod_{1 \leq j \leq N}\left|u_{j}\right|^{p_{j}}
$$

and

$$
\|\nabla F(u)\|=|F(u)| \sqrt{\sum_{1 \leq j \leq N} p_{j}^{2}\left|u_{j}\right|^{-2}}
$$

whenever $\prod_{1 \leq j \leq N}\left|u_{j}\right| \neq 0$. In particular

$$
\|\nabla F(u)\| \geq \gamma|F(u)| \sum_{1 \leq j \leq N}\left|u_{j}\right|^{-1}
$$

with

$$
\gamma=\frac{1}{\sqrt{N}} \inf _{1 \leq j \leq N} p_{j}>0 .
$$

Theorem 3.1. Let $F$ be given by (3.1). Then $F$ satisfies inequality (0.4) with $\theta=1 / d$.

Proof. We claim that

$$
\sum_{1 \leq j \leq N}\left|u_{j}\right|^{-1} \geq|F(u)|^{-1 / d}=\prod_{1 \leq j \leq N}\left|u_{j}\right|^{-p_{j} / d} .
$$


It is clear that (3.3) and (3.4) imply the result. The inequality (3.4) is a consequence of the following lemma, applied with $x_{j}=\left|u_{j}\right|^{-p_{j} / d}$ and

$$
\alpha_{j}=\frac{d}{p_{j}}=\frac{1}{p_{j}} \sum_{1 \leq j \leq N} p_{j} .
$$

Lemma 3.2. Let $\left\{\alpha_{j}\right\}_{1 \leq j \leq N}$ be a finite collection of exponents such that

$$
\forall j \in\{1, \ldots, N\}, \quad \alpha_{j}>1 \quad \text { and } \quad \sum_{1 \leq j \leq N} \frac{1}{\alpha_{j}}=1 .
$$

Then for any $N$-tuple $\left\{x_{j}\right\}_{1 \leq j \leq N}$ of non-negative numbers we have

$$
\prod_{1 \leq j \leq N} x_{j} \leq \sum_{1 \leq j \leq N} \frac{1}{\alpha_{j}} x_{j}^{\alpha_{j}} \leq \sum_{1 \leq j \leq N} x_{j}^{\alpha_{j}}
$$

Proof. This is an immediate consequence of concavity of the logarithm function on $[0, \infty)$.

4. Optimality and diagonal solutions. In this section, we study the optimality of the rate of convergence to stationary solutions given by the Łojasiewicz inequality. First of all we need to know something about the critical points of $F$. As a general remark we have

Proposition 4.1. Any critical point a of $F$ satisfies $F(a)=0$.

Proposition 4.1 is an immediate consequence of Proposition 1.1 and implies that convergence to equilibrium, when a Łojasiewicz inequality is satisfied, happens at least at the rate given by the exponent $\theta$ through inequality (0.5). In order to study the optimality of the rate of convergence, we introduce

Definition 4.2. A solution $u=\left(u_{1}, \ldots, u_{N}\right)$ of $(0.1)$ is said to be a diagonal solution if we have

$$
\forall j \in\{1, \ldots, N\}, \forall t \geq 0, \quad u_{j}(t)=c_{j} z(t) .
$$

Proposition 4.3. Assume $d \geq 2$. If $u$ is a diagonal solution, then $z$ has a constant sign and satisfies

$$
z^{\prime}(t)=C|z(t)|^{d-1}
$$

for some real constant $C$, the case $C=0$ corresponding to constant solutions.

Proof. The proposition is only of interest if not all constants $c_{j}$ are zero. Assuming for instance $c_{1} \neq 0$, we have

$$
z^{\prime}=c_{1}^{-1} u_{1}^{\prime}=-c_{1}^{-1} \partial_{1} F\left(c_{1} z, \ldots, c_{N} z\right)
$$


It is easy to deduce, since we assumed $d \geq 2$, that $z$ does not change sign on its maximal existence interval and then for some $\varepsilon \in\{1,-1\}$,

$$
z^{\prime}=-c_{1}^{-1} \partial_{1} F\left(\varepsilon c_{1}, \ldots, \varepsilon c_{N}\right)|z(t)|^{d-1} .
$$

COROLlary 4.4. If $u$ is a non-constant diagonal solution, then either it blows up at infinity or in finite time, or it is global and converges to 0 at infinity, exponentially fast if $d=2$, like $t^{-1 /(d-2)}$ if $d>2$.

Especially interesting is the case of generalized monomials. For instance we have the following complete description in the case of a positive generalized monomial function:

TheOREM 4.5. Let $F$ be given by

$$
F(u)=F\left(u_{1}, \ldots, u_{N}\right)=c \prod_{1 \leq j \leq N}\left|u_{j}\right|^{p_{j}}
$$

where $c>0$ and $p_{j} \geq 2$ for all $j \in\{1, \ldots, N\}$. Then the system (0.1) has a one-parameter family of diagonal solutions, characterized by the condition

$$
\exists \lambda \in \mathbb{R}, \forall j \in\{1, \ldots, N\}, \quad u_{j}(0)=(-1)^{m_{j}} \lambda \sqrt{p_{j}} .
$$

These solutions are the only ones tending to 0 as $t \rightarrow \infty$. In addition, assuming $d>2$, convergence to equilibrium of all other solutions occurs at a higher rate than $t^{-1 /(d-2)}$.

Proof. Let

$$
c_{j}=\sqrt{p_{j}}, \quad C=F\left(c_{1}, \ldots, c_{N}\right)
$$

and let $z$ be any positive solution of

$$
z^{\prime}+C z^{d-1}=0 .
$$

Setting $u_{j}(t)=c_{j} z(t)$ we have $u_{j}^{\prime}(t)=c_{j} z^{\prime}(t)=-c_{j} C z^{d-1}$, while on the other hand

$$
\begin{aligned}
\partial_{j} F\left(u_{1}, \ldots, u_{N}\right) & =\partial_{j} F\left(c_{1} z, \ldots, c_{N} z\right)=p_{j} c_{j}^{-1} z^{-1} F\left(c_{1} z, \ldots, c_{N} z\right) \\
& =c_{j} C z^{d-1} .
\end{aligned}
$$

It follows that $u=\left(u_{1}, \ldots, u_{N}\right)$ is a diagonal solution with

$$
u(0)=\left\{\sqrt{p_{j}} z(0)\right\}_{1 \leq j \leq N} .
$$

Since $z(0)$ can be an arbitrary positive number, this takes care of the part of the family corresponding to the positive sign for all components. The other cases follow by observing that each partial derivative $\partial_{j} F$ is odd with respect to $z_{j}$ and even in the other components. To show the second part of the theorem we observe that due to the special form of $F$ we have

$$
u_{j} u_{j}^{\prime}+p_{j} F(u)=0 .
$$


This yields the conservation laws

$$
\forall t \geq 0, \quad p_{i} u_{j}^{2}(t)-p_{j} u_{i}^{2}(t)=p_{i} u_{j}^{2}(0)-p_{j} u_{i}^{2}(0),
$$

giving in particular that any solution $u$ tending to 0 at infinity satisfies

$$
\forall t \geq 0, \quad p_{i} u_{j}^{2}(t)-p_{j} u_{i}^{2}(t)=0 .
$$

Using these identities we see that if $u_{i}\left(t_{0}\right)=0$ for some index $i$, then $u_{j}\left(t_{0}\right)=0$ for all $j$ and then $u \equiv 0$. Otherwise $u_{j} / u_{i}$ is constant along the trajectory, hence we have a diagonal solution. If $u$ is a non-diagonal solution, as a consequence of Theorem 3.1 we know that $u(t)$ converges at infinity. Some of the components tend to non-zero constants, and by Proposition 4.1 some other components tend to 0 . Assuming for instance $u_{1}(t) \rightarrow 0$, either $u_{1} \equiv 0$ and $u$ is constant, or the identity

$$
p_{1} u_{j}^{2}(t)-p_{j} u_{1}^{2}(t)=\gamma_{j} \geq 0
$$

shows two things:

$$
\begin{aligned}
& \text { 1) } u_{j}(t) \rightarrow 0 \Rightarrow u_{j}(t)=\varepsilon \sqrt{\frac{p_{j}}{p_{1}}} u_{1}(t), \varepsilon \in\{1,-1\}, \\
& \text { 2) } \gamma_{j}>0 \Rightarrow\left|\sqrt{p_{1}} u_{j}(t)-\varepsilon \sqrt{\gamma_{j}}\right| \sim k_{j} u_{1}^{2}(t), \varepsilon \in\{1,-1\} .
\end{aligned}
$$

Then it follows easily that either $u_{1}(t)$ tends exponentially to 0 , or

$$
\left|u_{1}(t)\right| \leq C t^{-1 /\left(p_{1}+\cdots+p_{r}-2\right)}
$$

if $u_{1}, \ldots, u_{r}$ are the components tending to 0 (a suitable reordering allows that). But now clearly $p_{1}+\cdots+p_{r}<d$. The result follows easily since the components not tending to 0 tend to their limits even faster (like $u_{1}^{2}(t)$ ).

REMARK 4.6. Theorem 4.5 shows that the global Łojasiewicz inequality gives a non-optimal rate of convergence to equilibrium for most of the solutions to (0.1) in this case. This is natural since for a solution tending to a non-zero limit $a$, the relevant inequality in the neighbourhood of $a$ is stronger (it involves only the components of the solution tending to 0 ), and the calculations above strongly suggest that we have exactly the right exponent.

5. Final remarks. First of all we give a more detailed assertion about the regularity of a positively homogeneous function $F$ at the origin:

Proposition 5.1. Let $F \in C^{1}\left(\mathbb{R}^{N}\right)$ be positively homogeneous of degree $d \in \mathbb{N}, d>1$, such that $F \in C^{d}(\Sigma)$. Then

(i) $F \in C^{d-1}\left(\mathbb{R}^{N}\right)$ with all partial derivatives of order $d$ in $L^{\infty}\left(\mathbb{R}^{N}\right)$.

(ii) $F \in C^{d}\left(\mathbb{R}^{N}\right)$ if and only if $F$ is a homogeneous polynomial on $\mathbb{R}^{N}$.

Proof. Clearly $F$ is $C^{d-1}$ with all partial derivatives up to order $d-1$ vanishing at 0 . Moreover all partial derivatives of order $d$, defined and con- 
tinuous on $\mathbb{R}^{N}-\{0\}$, are constant on each ray emanating from the origin. Being bounded on $\Sigma$, they are bounded globally. In addition, the existence of a limit at 0 imposes that all partial derivatives of order $d$ are constant on $\Sigma$ and therefore on $\mathbb{R}^{N}$. This obviously implies (ii).

Next we give more detailed results for some homogeneous functions which do not satisfy the Łojasiewicz inequality for the maximal value of $\theta$ :

Proposition 5.2. Let $F \in C^{d-1} \cap W^{d, \infty}\left(\mathbb{R}^{2}\right)$ be defined for some $d \in \mathbb{N}^{*}$, $p>0, \varepsilon \in\{0,1\}$ by

$$
F(x, y)=\frac{\left|x^{p+d}\right|(\operatorname{sgn} x)^{\varepsilon}}{\left(x^{2}+y^{2}\right)^{p / 2}} .
$$

Then $F$ satisfies the Lojasiewicz inequality near 0 for $\theta=1 /(p+d)$ and for no larger value of $\theta$.

The proof of Proposition 5.2 relies on the following lemma:

Lemma 5.3. Let $F \in C^{1}\left(\mathbb{R}^{2}\right)$. In order for $F$ to satisfy the Eojasiewicz inequality near 0 for some $\theta \leq 1 / d$, it is necessary and sufficient, with the notation of Section 2, that there exist $\alpha, C>0$ for which

$$
|f(\phi)| \leq \alpha \Rightarrow|f(\phi)|^{1-\theta} \leq C\left|f^{\prime}(\phi)\right| .
$$

Proof. Using the notation of Section 2 we have

$$
F(r, \phi)=r^{d} f(\phi), \quad\|\nabla F(r, \phi)\|=r^{d-1}\left(d^{2} f^{2}(\phi)+f^{\prime 2}(\phi)\right)^{1 / 2} .
$$

In order for $F$ to satisfy (0.4) in some sphere around 0 where $r$ is a small constant, it is necessary that for some constant $C>0$,

$$
|f(\phi)|^{1-\theta} \leq C\left(|f(\phi)|+\left|f^{\prime}(\phi)\right|\right) .
$$

Hence (5.2) must be fulfilled. Conversely, for any $\theta>0$, (5.2) implies (5.3). In addition if $\theta \leq 1 / d$ we have $r^{d}(1-\theta) \leq r^{d-1}$ for $r \leq 1$. The conclusion follows immediately.

Proof of Proposition 5.2. Since we have

$$
f(\phi)=\sin ^{p+d} \phi
$$

the result is an immediate consequence of condition (5.2).

Proposition 5.4. Let $F \in C^{1} \cap W^{2, \infty}\left(\mathbb{R}^{2}\right)$ be defined for some $p \geq 0$ by

$$
F(x, y)=\left(x^{2}+y^{2}\right)^{p} \exp \left(-\frac{x^{2}+y^{2}}{x^{2}}\right) .
$$

Then, in the neighbourhood of $0, F$ does not satisfy the Lojasiewicz inequality for any $\theta>0$. 
Proof. Since we have

$$
f(\phi)=\exp \left(-\frac{1}{\sin ^{2} \phi}\right)
$$

the result is an immediate consequence of condition (5.2).

REMARK 5.5. Failure to satisfy the Łojasiewicz condition may result in non-convergence (cf. e.g. [11]).

\section{References}

[1] E. Feireisl and F. Simondon, Convergence for semilinear degenerate parabolic equations in several space dimensions, J. Dynam. Differential Equations 12 (2000), 647673.

[2] A. Haraux and M. A. Jendoubi, Convergence of solutions of second-order gradientlike systems with analytic nonlinearities, J. Differential Equations 144 (1998), 313320 .

[3] - - - Convergence of bounded weak solutions of the wave equation with linear dissipation and analytic nonlinearity, Calc. Var. 9 (1999), 95-124.

[4] -, 一, Decay estimates to equilibrium for some evolution equations with an analytic nonlinearity, Asymptotic Anal. 26 (2001), 21-36.

[5] A. Haraux, M. A. Jendoubi and O. Kavian, Rate of decay to equilibrium in some semilinear parabolic equations, to appear.

[6] M. A. Jendoubi, A simple unified approch to some convergence theorems of L. Simon, J. Funct. Anal. 153 (1998), 187-202.

[7] -, Convergence of global and bounded solutions of the wave equation with linear dissipation and analytic nonlinearity, J. Differential Equations 144 (1998), 302-312.

[8] S. Łojasiewicz, Ensembles semi-analytiques, I.H.E.S. notes, 1965.

[9] -, Une propriété topologique des sous ensembles analytiques réels, in: Les équations aux dérivées partielles (Paris, 1962), Éd. CNRS, Paris, 1963, 87-89.

[10] - Sur les trajectoires du gradient d'une fonction analytique, in: Seminari di geometria, Bologna (1982-83), Univ. Stud. Bologna, Bologna, 1984, 115-117.

[11] J. Palis and W. de Melo, Geometric Theory of Dynamical Systems: An Introduction, Springer, New York, 1982.

[12] L. Simon, Asymptotics for a class of non-linear evolution equations, with applications to geometric problems, Ann. of Math. 118 (1983), 525-571.

Laboratoire Jacques-Louis Lions

Université Pierre et Marie Curie

Boîte courrier 187

75252 Paris Cedex 05, France

E-mail: haraux@ann.jussieu.fr

Reçu par la Rédaction le 19.5.2004

Révisé le 20.10.2005 